\title{
Reconstruction of oviraptorid clutches illuminates their unique nesting biology
}

Tzu-Ruei Yang, Jasmina Wiemann, Li Xu, Yen-Nien Cheng, Xiao-Chun Wu, and P. Martin Sander Acta Palaeontologica Polonica 64 (3), 2019: 581-596 doi:https://doi.org/10.4202/app.00497.2018

Oviraptorosaurs, a group of non-avian theropod dinosaurs from the Cretaceous of Asia and North America, left behind the most abundant and informative fossil evidence of dinosaur reproductive biology. Previous studies had suggested that oviraptorosaur reproductive biology represents an intermediate stage and exhibited unique modern avian traits. For instance, the adult-associated clutches were predominantly considered as evidence for brooding/thermoregulatory contact incubation (TCI) behaviors, whereas the hypotheses of laying or protection were neglected. Despite numerous oviraptorid egg clutches uncovered from China and Mongolia, their nest architecture and clutch arrangement were rarely investigated in detail. Here we present a comprehensive reconstruction of an oviraptorid clutch based on five new oviraptorid clutches from Jiangxi Province, China. A detailed examination of the new clutches reveals a partially-open oviraptorid nest that contains 3-4 rings of paired eggs (more than 15 pairs total) whose blunt end points toward the center devoid of eggs at an angle of 35-40. . Our detailed three-dimensional reconstruction indicates that the oviraptorid clutch has a unique architecture unknown from extant bird clutches, implying an apomorphic nesting mode. Such a unique nest architecture further contradicts the TCI hypothesis in oviraptorids, hindering sufficient heat transfer to the inner (lower) ring(s) of eggs. Moreover, the size of the new oviraptorid clutches ( $>30$ eggs) is significantly larger than that of the adult-associated clutches ( 